\title{
Family Care giving in Duchenne Muscular Dystrophy: Is there a gender difference?
}

\author{
${ }^{1}$ Priya Treesa Thomas PhD, ${ }^{2}$ Prakashi Rajaram PhD, ${ }^{3}$ Nalini A. DM (Neuro) \\ ${ }^{I}$ Psychiatric Social Worker, Dept of Psychiatric Social Work, NIMHANS, Bangalore - 560029 \\ ${ }^{2}$ Additional Professor, Dept of Psychiatric Social Work, NIMHANS, Bangalore - 560029 \\ ${ }^{3}$ Professor, Dept of Neurology, NIMHANS, Bangalore - 560029
}

\begin{abstract}
Families of children with a Duchenne Muscular Dystrophy go through significant challenges in dealing with the condition. Few studies have examined the situations especially in the socio-cultural scenario that is unique to India, looking into the gender difference in the challenges. Hence, the aim of the present study was to identify the gender difference in psychosocial challenges in care giving for caregivers of children with Duchenne Muscular Dystrophy (DMD). A cross sectional descriptive study was performed among the caregivers of 60 children with DMD (All Males, Mean age 8.60; SD 1.76) who were attending the neuromuscular disorders clinic of a national tertiary referral center for Neurological disorders. All the children had a diagnosis of DMD confirmed either by genetic analysis or absence of dystrophin staining on muscle Immunohistochemistry. The caregivers included fathers (32), mothers (24) and other relatives (4), with mean age 35.20; SD 6.61.The knowledge and attitude, caregiver wellbeing, burden and coping patterns were assessed using structured interview schedule. The findings showed that while there was no significant difference among the parents of children with DMD on knowledge and attitude, significant differences were found in the experience of family burden and coping. The study concluded that caregivers of children with DMD are expected to benefit from psychosocial intervention to address their inadequate knowledge, attitude and burden, in keeping with the gender difference in caregiving, and to help them develop coping skills and meet their needs as well as their children's needs.

Contact information of corresponding author: priyathomasat@gmail.com
\end{abstract}

\section{Introduction}

Duchenne Muscular Dystrophy (DMD) is marked by progressive weakness and degeneration of the skeletal or voluntary muscles. This is the most common form of Muscular Dystrophies that affect 1 in 3500 live male births (Emery, A. E., 1991). It is inherited in X linked pattern. Hence, women are carriers and affected male children manifest the disease. The disease often becomes noticeable in early childhood as delay in walking, later symptoms including general muscle weakness and wasting. It affects pelvis, upper arms and upper legs, eventually involves all voluntary muscles. The child with DMD is non ambulatory by 10-12 years and death occurs by 18-20 years. There is a significant advancement in its management, including treatment with corticosteroids and the use of intermittent positive pressure ventilation which provided improvements in function, ambulation, quality of life and life expectancy (Yiu and Kornberg, 2008). However, novel therapies are yet to find in order to cure this disease. Thus DMD continues to be $100 \%$ fatal disease with significant psychosocial implications on the patients and families.

When diagnosis of DMD takes place, the family is warned that DMD is an inherited disorder and that the sisters and cousins of the affected boy have a high risk of being carriers. The diagnosis presents parents with many challenges. Mothers, in particular, have the additional burden of realizing that they may be the unsuspecting carriers of the mutation responsible for the disorder. Therefore, as the disease progresses, mothers may develop an attitude of self-blame (Rubin, 1987). Both parents may react with feelings of disbelief, denial, anger, anguish, anxiety, guilt (Buchanan et al., 1979; Rubin, 1987; DMD Forum, 2001) fear, confusion, powerlessness, rejection (DMD Forum, 2001), and parent/child-related stress (Beresford, 1994).

Tomiak et al. (2007) conducted a qualitative enquiry into the gender specific differences in the psychosocial adjustment of parents of a child with DMD. The research focused on the key psychological aspects of the process of adjustment to the illness of their child, such as the family and spousal relationship, daily life, emotions, career, spirituality and coping strategies. The study revealed that there are significant differences in the initial reaction and coping styles between mothers and fathers. The authors describe the lived experience of parents of a child with DMD with specific attention to the internal dynamic of the relationship between father and mother. This internal dynamic is highly dependent on the respective roles of primary and secondary caregiver: the primary caregiver role is usually assumed by the mother, with the father providing a supportive role. In the context of the chronic illness, the traditional stereotypes seemed to be regenerated, with the mother providing daily care and the major emotional support for the child and the family. The father's major 
contributions are in overseeing the overall functioning of the family unit and in the moral and psychological domains. Gravelle (1997) found that mothers were the primary caregivers in both direct and indirect care giving. In her qualitative inquiry into the care giving experience of parents of children with DMD, she found that mothers readily assumed the care giving role as a natural extension of their mothering role. Gray (2003) who conducted a qualitative enquiry into gender and coping among mothers of children with a chronic disorder found that the existence of the child's disability may return the family to the traditional pattern of gender relations.

Nereo, Fee and Hilton (2003) examined parental stress and its predictors in mothers of 112 boys with DMD and comparisons were made with mothers of healthy children, children with cerebral palsy, siblings of boys with DMD and longitudinally. They found that presence of problem child behavior was the consistent predictor for maternal stress. They further concluded that stress in mothers of boys with DMD is elevated, possibly due to increased problem behaviors, particularly in social interactions, rather than due to the physical demands of the disease alone.

In India, disability is still viewed in terms of tragedy with fatalistic attitudes and external dependence by the families of disabled children (Dalal and Pandey, 1999). There is a very strong belief in the metaphysical causation. Cultural beliefs about disability are very important and are crucial in family's perception about the disability and it determines how family act in terms of treatment, prevention and rehabilitation. One factor which functions in this context is the belief in the theory of Karma. It is often used to explain major life events, including the occurrence of disability (Sen, 1988). Parents of Indian children with DMD reported a bimodal peak in stress wherein one smaller stress peaks was observed at the time of the diagnosis and the second higher one at the time when boys become immobile. The parents in this cohort tended to use denial as a coping strategy for unusually longer periods of time and even in acceptance they experienced chronic guilt and helplessness (Nadkarni et al., 2008).

The present study seeks to explore the emotional, social and economic strain of the family members undergo while caring for a child with DMD, and to find out if there is the difference in the caregiving stress among the mothers and fathers. The way each parent cope with caregiving challenges poses a major influence the coping of the other parents and other family members, including the affected child. In spite of this, studies that focus on parental adjustment to DMD in the socio-cultural context that is unique to India have been few and far in between. Given this context, a cross sectional study was conducted to examine the psychosocial challenges of among the parents of children with Muscular Dystrophy. The specific objectives were to identify the burden faced by the parents and to find out the differences in burden experienced by the individual parent. From a clinical social work perspective, it is critical to understand the areas of caregiving needs of mother and father of the child, family burden experience and coping strategies used to adapt to the caregiving challenges and situations. Such insights are expected to help to design appropriate psychosocial interventions aiming at psychosocial wellbeing of caregiving parents thereby improve the quality of care.

\section{Method}

The study participants were the caregivers of children diagnosed to have Duchenne Muscular Dystrophy (DMD) following a detailed clinical examination, muscle biopsy and/ or genetic analysis, as per the diagnostic criteria and met the eligibility criteria for participation.

\section{Participants}

All 60 caregivers of children with DMD who sought healthcare in a tertiary referral centre for neurology at National Institute of Mental Health and Neuro Sciences situated in the city of Bangalore. The study participants who met the eligibility criteria were prospectively selected from 2007 to 2009. All participants were attending the Neuromuscular Disorders Clinic, a special clinic held as part of neurology outpatient department. The caregivers were both parents or in rare cases, a significant family member.

\section{Eligibility criteria}

The eligibility criteria were a diagnosis of DMD, in a child aged between 4 to 13 years. The caregivers with active psychopathology were excluded from the study.

\section{Measurements}

A structured interview schedule was developed that contained selected socio demographic variables pertaining to the patient and the caregiver information regarding the diagnosis and treatment..

Knowledge and Attitude towards Muscular Dystrophy: For the current study, a structured interview schedule was developed. It consists of 35 items measuring knowledge and attitude of caregivers towards Muscular Dystrophy on five sub-domains viz., etiology and nature of illness, symptoms, management of condition, incidence and prevalence and diagnosis and prognosis. In the schedule, the responses for 15 items 
were scored as 'one point' for 'true' and 'zero point' for 'false' and 'don't know.' These items were numbered $2,8,9,11,12,17,20,23,24,26,28,32,33,34$ and 35 . Whereas, the remaining 20 items numbered 1, 3, 4, 5, 6, $7,10,13,14,15,16,18,19,21,22,25,27,29,30$ and 31 reverse scored, with 'one point' being 'false' and 'zero point' for 'true' and 'don't know'. The obtainable scores on the schedule ranged from 1-35. Thus unit weight-age method was adopted. The items on this schedule were developed through an extensive systematic literature review, interviews with families of patients with Muscular Dystrophy as well as discussions with experts in the field. Further, the schedule was face validated by a group of experts. But no effort was made to establish psychometric properties such as reliability and validity for this interview schedule.

Family burden assessment scale (Pai and Kapur, 1981): This scale consists of 25 items spread across seven sub-domains of family burden viz., financial burden (6 items), disruption in family routine (5 items), family leisure (5 items), family interaction ( 5 items), physical health of others ( 2 items) (than the patient) effects of mental health of others ( 2 items) and subjective burden (one item). Though it was primarily developed for measuring the burden experienced by the families of persons with schizophrenia, this has been widely used in other clinical conditions such as to assess the burden among families of neurotic patients, patients with alcohol abuse, mental retardation, chronic physical illness such as stroke. No reliability score was observed below 0.78 while validity was established by studying the correlations between burden assessed by raters and subjective burden assessed by relatives at $\mathrm{r}=0.72$.

Coping with DMD: The COPE Inventory (Carver et al 1989) is a 60 items inventory designed to measure a broad range of coping responses and coping patterns adopted by the families. The coping is measured on 15 factors reflecting active versus avoidant coping strategies. Ratings are made on a 4-point Likert type scale that ranges from "I (usually) don't do this at all=1" to "I (usually) do this a lot=4". Its factors are active coping, planning, seeking instrumental and emotional social supports, suppression of competing activities, religion, positive reinterpretation and growth, restraint coping, resignation/acceptance, venting emotions, denial, mental disengagement, behavioural disengagement, alcohol or drug use and humor. The inventory has good psychometric properties with alphas ranging from .45 to .92 , test-retest reliabilities ranging from .46 to .86 , and strong evidence of discriminant and convergent validity, with constructs such as hardiness, optimism, control and self-esteem.

\section{Ethical considerations}

Informed consent was obtained from each study participant before participating in the study. Further, the study protocol obtained ethical clearance from the institutional ethics committee of National Institute of Mental Health \& Neuro Sciences, Bangalore (NIMHANS).

\section{Statistical analysis}

In the current study, data analysis progressed from descriptive analysis viz., frequencies, percentage, measures of central tendencies and dispersion to test of differences using mean, SD, independent sample t' test.

\section{Sociodemographic and illness characteristics}

Table-1 shows the socio-demographic and illness characteristics of children with DMD. The age of the children ranged from 6 years to 13 years with a mean of 8.60 and SD of 1.76. Out of 60 children, ten of them $(17.7 \%)$ reported positive family history of DMD and five of them $(8.3 \%)$ had an affected family member alive at the time of interview. Most of the children were attending schools (88.3\%) among whom 48 children were attending regular schools and five of them were attending special schools. Seven children dropped out from schools due to progressive disability coupled with lack of facilities made it difficult for them and to their parents to continue with schooling. Most children (60\%) were depended on caregivers for toilet needs and ambulation, especially climbing stairs and getting up from squatting. All children reported difficulty in getting up from squatting position at the time of initial need assessment which is one of the primary clinical characteristic of DMD (Guberman, 1994). Further, most children experienced difficulties in climbing stairs (93.3\%), in fast walk or run $(98.3 \%)$ and calf hypertrophy $(96.7 \%)$.

Table 1: Illness details of the children with DMD

\begin{tabular}{|c|l|}
\hline Duration of illness & $\mathrm{N}(\%)$ \\
\hline$\leq 2.5$ years & $12(20.0)$ \\
$\geq 4.6$ years & $37(61.7)$ \\
\hline$\leq 5$ months & $11(18.3)$ \\
\hline Duration of diagnosis & \\
\hline 28 months & $6(10)$ \\
$\geq 29$ months & $39(65)$ \\
\hline Treatment from other healthcare disciplines & $15(25)$ \\
prior to allopathic treatment & \\
\hline
\end{tabular}


Family Care giving in Duchenne Muscular Dystrophy: Is there a gender difference?

\begin{tabular}{|l|l|}
\hline \multicolumn{1}{|c|}{ Yes } & \\
\hline No & $9(15)$ \\
& $51(85)$ \\
\hline Activities of Daily Living & \\
\hline Independent & $24(40)$ \\
Partially independent & $36(60)$ \\
\hline
\end{tabular}

Table 2 shows the caregivers' socio-demographic characteristics. In caregivers, 32 (53.3\%) of them were fathers, 24 of them were mothers (40\%) and significant other relatives constituted 4 persons $(6.7 \%)$. Most of the families were from the rural areas $(73.3 \%)$.

Table 2: Demographic details of the caregivers

\begin{tabular}{|l|l|}
\hline Caregiver details & $\mathbf{N}(\%)$ \\
\hline Age & \\
\hline 29 years \& Below & $6(10)$ \\
$30-38$ years & $41(68.3)$ \\
39 \& Above & $13(21.7)$ \\
& \\
& \\
\hline Sex & \\
\hline Male & $35(58.3)$ \\
Female & $25(41.7)$ \\
\hline Family history of DMD & \\
\hline Present & $10(17.7)$ \\
Absent & $50(83.3)$ \\
\hline
\end{tabular}

\section{Knowledge, attitude, wellbeing and Family burden}

\section{Results}

In the present study, there was no significant difference between men and women in knowledge, attitude and care giver wellbeing (Table 4). Women caregivers reported higher family burden than the men ( $t$ $=3.07 ; \mathrm{p}=0.003)$. This may be because women engage more frequently and consistently in direct caregiving activities in an informal family caregiving context than men.

Table 4: Caregiver knowledge, attitude, wellbeing and family burden among men and women

\begin{tabular}{|l|l|l|l|l|}
\hline Sl no & Factors & Men M(SD) & Women M(SD) & 't' value \\
\hline 1 & $\begin{array}{l}\text { Knowledge about Muscular } \\
\text { Dystrophy }\end{array}$ & $26.37(19.46)$ & $22.97(13.39)$ & 0.75 \\
\hline 2 & Attitude & $74.56(5.69)$ & $74.98(3.84)$ & -0.33 \\
\hline 3 & Caregiver Wellbeing & $51.21(5.32)$ & $48.80(5.19)$ & 1.75 \\
\hline 4 & Family burden & $35.94(12.33)$ & $47.12(15.88)$ & $3.07^{*}$ \\
\hline
\end{tabular}

*significant at $\mathrm{p}<0.05$

\section{Caregivers' coping strategies:}

The most common coping strategy employed by the caregivers was religious coping (Mean=12.75; $\mathrm{SD}=2.99$ ) and focus on and venting of emotions (Mean=11.77; $\mathrm{SD}=3.32$ ). Social support, both instrumental (Mean=11.42; $\mathrm{SD}=3.69$ ) and emotional (Mean=10.50; $\mathrm{SD}=2.99$ ) were frequently used by the caregivers. Humour was the least frequently used coping strategy (Mean=4.02; $\mathrm{SD}=0.13$ ), followed by positive reinterpretation and growth (Mean=4.68; $\mathrm{SD}=1.24$ ). Religious coping significantly differed between male and female caregivers $(t=3.94 ; \mathrm{p}<0.001)$ wherein female caregivers more frequently used religious coping than male caregivers. Consistently, focus on and venting of emotions was also significantly differed between male and female caregivers $(\mathrm{t}=4.79 ; \mathrm{p}<0.001)$ while female caregivers used it more frequently than the other. However, substance use was found to be high among the men than women caregivers $(t=2.89 ; \mathrm{p}=0.005)$.

Table 5: coping strategies among the men and women

\begin{tabular}{|l|l|l|l|l|}
\hline \multirow{2}{*}{ SL NO } & \multirow{2}{*}{ FACTORS } & Male (35) & Female (25) & 't' value \\
\cline { 3 - 5 } & & Mean (SD) & Mean (SD) & \\
\hline 1 & Positive reinterpretation and growth & $4.77(1.49)$ & $4.56(0.77)$ & 0.65 \\
\hline 2 & Mental dis engagement & $7.8(3.99)$ & $6.84(2.93)$ & 1.02 \\
\hline 3 & Focus on \& venting of emotions & $10.29(2.86)$ & $13.84(2.81)$ & $-4.79 * * *$ \\
\hline 4 & Use of instrumental social support & $11.14(3.79)$ & $11.8(3.59)$ & -0.68 \\
\hline 5 & Active coping & $7.37(2.65)$ & $6.84(2.29)$ & 0.81 \\
\hline 6 & Denial & $6.86(3.93)$ & $6.52(2.82)$ & 0.37 \\
\hline 7 & Religious coping & $11.6(3.03)$ & $14.36(2.08)$ & $3.94 * * *$ \\
\hline 8 & Humor & $4.03(0.17)$ & $4.00(0)$ & 0.84 \\
\hline
\end{tabular}


Family Care giving in Duchenne Muscular Dystrophy: Is there a gender difference?

\begin{tabular}{|l|l|l|l|l|}
9 & Behavioual disengagement & $9.37(4.35)$ & $7.28(4.41)$ & 1.83 \\
\hline 10 & Restraint & $5.8(1.53)$ & $5.40(1.04)$ & 1.13 \\
\hline 11 & Use of emotional social support & $10.17(2.99)$ & $10.96(2.78)$ & -1.04 \\
\hline 12 & Substance use & $6.06(2.69)$ & $4.44(0.87)$ & $2.89 *$ \\
\hline 13 & Acceptance & $9.34(3.54)$ & $6.03(4.34)$ & -0.99 \\
\hline 14 & Suppression of competing activities & $6.03(2.44)$ & $5.8(2.18)$ & 0.37 \\
\hline 15 & Planning & $6.83(2.47)$ & $5.96(1.34)$ & 1.59 \\
\hline
\end{tabular}

*significant at $\mathrm{p}<0.05$

$* * *$ significant at $\mathrm{p}<0.001$

\section{Discussion}

Interestingly, most of the caregivers were males in the present study, which is a departure from the caregiving literature that equates care giving to the role of a mother (Tomiak , 2007), and might represent a caregiving pattern characteristic to the Indian culture. Plausible explanations may be that either the cultural factors that restrict the mobility of women outside homes or due to inherent lack of confidence that results in not venturing to a formal setting such as the hospital, the father of the child is the family member who usually accompanies the child to the follow up. Further, increasing disability of the child makes it difficult for the mothers to help the children in terms of transportation to outside by themselves. Finally, since many of the caregivers were in the reproductive age group, mothers often have to care for younger siblings.

The present study found mothers, and other female caregivers to experience greater levels of stress than the fathers, and other male caregivers. This is in concurrence with the caregiving literature that state women to have more caregiving stress than men. (Dellve, 2006; Tomiak, 2007; Gray, 2003; Rubin, 1987)

Significant differences were observed among the fathers and mothers with regard to the coping strategies of focus on and venting of emotions $(\mathrm{P}<0.001)$, religious coping $(\mathrm{P}<0.001)$ and substance abuse $(\mathrm{P}<0.05)$. Mothers and other female caregivers tended to utilize the emotional ventilation and religious coping as a coping strategy more often than the fathers and male caregivers. While men in general reported more knowledge and wellbeing in different domains, this difference was not found to be statistically significant. Mothers had a more positive attitude $(86.65 \pm 3.08)$ regarding the disease than the fathers. This can probably be explained in terms of the direct care giving role assumed by the female caregivers as a natural extension of the mothering role.

Men and women differed on their family burden significantly $(p<0.01)$ with the female respondents having significantly more family burden. Further, mothers reported more burden in terms of disruption of family interaction than the fathers. Significant difference was observed between fathers and mothers on disruption of family leisure, with mothers reporting more burden in disruption of family leisure than the fathers. These findings are in line with those of Nereo et al (2003), who examined parental stress and its predictors in mothers of 112 boys with Duchenne muscular dystrophy (DMD). They concluded that stress in mothers of boys with DMD is elevated, possibly due to increased problem behaviours, particularly in social interactions, rather than due to the physical demands of the disease alone.

One of the areas that was reported to be more significant in burden was the financial implications associated with the disease and caregiving. An analysis of the circumstances contributing to increased risk of impoverishment for young children with disabilities by Parish and Cloud (2006) had concluded that among other factors, elevated costs of raising children with disabilities, difficulty in balancing parental, especially maternal employment and care giving responsibilities, unavailable or high cost child care and inadequate leave time to permit parents to meet their children's episodic care needs contribute to increased risk. The impact of the disease on the mental health of the caregivers was found to be moderately high, consistent with the findings that parents of children with DMD were more likely to suffer a major depressive episode (Abi Daoud, Dooley and Gordon, 2004).

As Samson et al (2009) reports, lived experience of caring for a child with Muscular Dystrophy goes much beyond the factors that could be brought out through quantitative method of inquiry. As in other studies dealing with similar population, sample size in the present study was low. The present study focused on the caregivers who accompanied the child with DMD to the hospital follow ups. In most cases, attempts to get continued access to the primary caregivers, mostly mothers were not possible because of the cultural and economic factors.

On the basis of the present study, the need for psychiatric social work services with the families with DMD through an approach informed by individual family needs and the family systems life cycle was made felt. Clinical Social Workers while working with the specific population like families of children with chronic disorders need to be guided by the evidence identified by similar research. Specific attention needs to be paid to the women caregivers, who are mostly mothers or maternal relatives in this case, and their special concerns need to be addressed in the intervention. The effectiveness and need of a close team work in health care is to be recognized and effective collaboration with other team members is essential in service delivery. The evidence 
generated by this study is helpful in planning direct social work services with specific populations with chronic childhood diseases. Training for social workers at the post graduate and pre-doctoral programmes should include end of life care issues and working with the patients and family members of chronically ill patients.

\section{References}

[1]. Abi Daoud, M., Dooley, J.M. and Gordon, K.E. (2004). Depression in parents of children with Duchenne Muscular Dystrophy. Pediatric Neurology, 31, 16-19.

[2]. Beresford, B. (1994). Resources and strategies: how parents cope with the care of a disabled child. Journal of Child Psychology and Psychiatry, 35, 171-209.

[3]. Boyer, F., Drame, M., Morrone, I., and Novella, J. L. (2006). Factors relating to carer burden for families of persons with muscular dystrophy. Journal of Rehabilitation Medicine, 38, 309-315.

[4]. Buchanan, D., LaBarbera, C., Roelofs, R., and Olson, W. (1979). Reactions of families to children with Duchenne muscular dystrophy. General Hospital Psychiatry, 1, 262-269.

[5]. Carver, C. S., Scheier, M. F., and Weintraub, J. K. (1989). Assessing coping strategies: A theoretically based approach. Journal of Personality and Social Psychology, 56, 267-283.

[6]. Dalal, A.K., Pandey, N. (1999). Cultural beliefs and Family care of the children with Disability.

[7]. Psychology and Developing Societies, 11, 55 - 75.

[8]. Dellve, L., Samuelsson, L., Tallborn, A., Fasth, A. \& Hallberg, L.R.M. (2006). Stress and wellbeing among parents of children with rare diseases: A Prospective Intervention study. Journal of Advanced Nursing, 53, 392 - 402

[9]. DMD Forum (2001) Guides for Parents - Parenting. Available at: http://www.dmdforum.org/guides/parenting.html (retrieved 5 March 2006).

[10]. Emery, A. E. (1991). Population frequencies of inherited neuromuscular diseases- a world survey. Neuromuscular Disorder, 1, 1929.

[11]. Gravelle, A. M. (1997). Caring for a child with a progressive illness during the complex chronic

[12]. phase: parents' experience of facing adversity. Journal of Advanced Nursing, 25, 738 - 745.

[13]. Gray, D. (2003). Gender and Coping: The parents of children with high functioning Autism. Social Science and Medicine, 56, 631 -642 .

[14]. Guberman, A. (1994). An Introduction to Clinical Neurology. Little Brown: London.

[15]. Holroyd, J., and Guthrie, D. (1979). Stress in families of children with neuromuscular disease. Journal of Clinical Psychology, 45, $734-739$.

[16]. Holroyd, J., and Guthrie, D. (1986). Family stress with chronic childhood illness: Cystic Fibrosis, Neuromuscular Disease, and renal disease. Journal of Clinical Psychology, 42, 552 - 561.

[17]. Kaslow, N. J., Collins, M.H., Brown, F., Baskin, M., Griffith, J., and Eckman, J.(2000). Efficacy of family psychoeducational intervention for pediatric sickle cell disease. Family Systems and Health, 18, 381-404.

[18]. Maddocks, I., Stern, L., and Parker, D. (1998). The Palliative Care of advanced MD and Spinal Muscular Atrophy. The Flinders University of South Australia: Adelaide.

[19]. Nadkarni, J., Dastur, R., Viswanathan, V., Gaitonde, P., and Khadilkar, S. (2008). Duchenne and Becker muscular dystrophies: An Indian update on genetics and rehabilitation. Neurology India, 56, $248-53$.

[20]. Nereo, N.E., Fee, R.J., and Hinton, V.J. (2003). Parental Stress in Mothers of Boys with Duchenne Muscular Dystrophy. Journal of Pediatric Psychology, 28, 473-484.

[21]. Pai, S., and Kapur, R. L. (1981). The burden on the family of a psychiatric patient: Development of an assessment scale. British Journal of Psychiatry, 38, 332-335.

[22]. Parish, S.L., and Cloud, J.M. (2006). Financial Wellbeing of Young children with disabilities and their families. Social Work, 51, $223-232$.

[23]. Rubin, S. (1987) The psychological impact of genetic disease. In: L. Charash, R. Lovelace, S.Wolf, A. Kutscher, D. Roye \& C. Leach (eds) Realities in Coping with Progressive Neuromuscular Diseases The Charles Press, Philadelphia, PA, USA. pp. $209-215$.

[24]. Samson, A., Tomiak, E.M., Demillo, J., Lavigne, R., Miles, S.A., Choquette, M.C., Chakraborty,

[25]. P.K., and Jacob, P. J. (2009). The lived experience of hope among parents of a child with Duchenne muscular dystrophy: percei ving the human being beyond the illness. Chronic Illness, 5, 103-114.

[26]. Sen, A. (1988). Psychosocial Integration of the Handicapped. Mittal Publications: New Delhi.

[27]. Tebb, S. (1995). An aid to empowering: A caregiver well-being scale. Health and Social Work, $20,87-92$.

[28]. Tomiak, E.M., Samson, A., Miles, S.A., Choquette, M.C., Chakraborty, P.K. and Jacob, P.J.

[29]. (2007). Gender- specific differences in the psychosocial adjustment of parents of a child with Duchenne Muscular Dystrophy (DMD). Qualitative Research Journal, 7, 2-21.

[30]. Webb, C. L. (2005). Parents' perspectives on coping with Duchenne muscular dystrophy. Child: Care, Health and Development, 31, 385-396.

[31]. Yiu, E., and Kornberg, A. (2008). Duchenne Muscular Dystrophy. Neurology India, 56, 236 - 247. 